\title{
XIAP expression is associated with pancreatic carcinoma outcome
}

\author{
SHENGMIAN LI ${ }^{1}$, JIANJIAN SUN $^{1}$, JIAN YANG $^{1}$, LAN ZHANG $^{1}$, LE WANG $^{1}$, \\ XIAOLING WANG $^{2}$ and ZHANJUN GUO ${ }^{1}$ \\ Departments of ${ }^{1}$ Gastroenterology and Hepatology and ${ }^{2}$ Pathology, The Fourth Hospital of Hebei Medical University, \\ Shijiazhuang, Hebei 050011, P.R. China
}

Received September 6, 2012; Accepted December 19, 2012

DOI: $10.3892 / \mathrm{mco} .2013 .58$

\begin{abstract}
Pancreatic carcinoma is an aggressive tumor that is resistant to treatment regimens. Although the X-linked inhibitor of apoptosis protein (XIAP) overexpression in pancreatic carcinoma cells has been previously reported, the aim of this study was to investigate the association of XIAP expression with pancreatic carcinoma outcome. Specimens were obtained from 54 patients who underwent resection for pancreatic cancer. Kaplan-Meier survival analysis was used to estimate post-operative survival. The results of this analysis revealed a significantly shorter overall survival time in patients with a high compared to those with a low XIAP expression $(\mathrm{P}=0.041)$. Results of the multivariate analysis revealed that XIAP expression was identified as an independent predictor for pancreatic carcinoma outcome [relative risk, 1.771; 95\% confidence interval (CI), 1.099-2.852; $\mathrm{P}=0.019]$. Moreover, XIAP levels were associated with pancreatic carcinoma characteristics, including tumor invasion status and histological grade. XIAP overexpression shortens the survival of pancreatic cancer patients probably by modifying their resistance to apoptosis and the proliferation capacity of pancreatic carcinoma cells. Thus, XIAP expression analysis can help in the identification of patient subgroups at high risk of a poor disease outcome.
\end{abstract}

\section{Introduction}

Pancreatic carcinoma is one of the most aggressive tumors and continues to constitute a major health problem. The overall 5 -year survival rate of pancreatic cancer patients remains poor $(<5 \%)$ despite treatment improvement $(1,2)$. The resistance of pancreatic carcinoma to aggressive treatment regimens represents a major challenge, whereas pancreatic carcinoma cell resistance to apoptosis contributes to treatment failure $(3,4)$.

Correspondence to: Dr Shengmian Li, Department of Gastroenterology and Hepatology, The Fourth Hospital of Hebei Medical University, 12 Jiankang Road, Shijiazhuang, Hebei 050011, P.R. China

E-mail: lishengmian@yahoo.com.cn

Key words: X-linked inhibitor of apoptosis protein, outcome, pancreatic carcinoma, apoptosis
Apoptosis is initiated by caspase activation in mitochondria (5). Resistance to apoptosis has been associated with the overexpression of extrinsic and intrinsic apoptosis signaling cascades (6). The X-linked inhibitor of apoptosis protein (XIAP) belongs to the inhibitor of apoptosis proteins (IAPs) family, a group of structurally related proteins inducing caspase inhibition that block apoptosis at the core of the apoptotic machinery by inhibiting caspase- $3,-7$ and -9 activation, thereby modulating resistance to apoptosis in pancreatic carcinoma (7).

Although not expressed in normal pancreatic ductal cells, XIAP overexpression is known to occur in pancreatic carcinoma cells, as noted in previous studies (7). The aim of this study was to investigate the association of resistance to apoptosis with disease survival by assessing the predictive power of XIAP expression on pancreatic carcinoma outcome.

\section{Materials and methods}

Tissue collection. In total, 54 patients (34 men and 20 women; age, 34-79 years) who underwent macroscopically curative resection for pancreatic carcinoma between 2003 and 2009 in The Fourth Hospital of Hebei Medical University (Shijiazhuang, China) were included in this study. Clinical data were collected for the patients, and histopathological and tumor-node-metastasis (TNM) classifications were defined in accordance with the International Union Against Cancer (UICC) Classification of 2002 (8). Vascular invasion or lymph node metastases and differentiated grade were also determined using hematoxylin and eosin (H\&E) staining. The specimens were fixed immediately in $10 \%$ formalin for immunostaining. The procedures were supervised and approved by the Human Tissue Research Committee of the hospital. Informed consent was obtained from the patients.

Measurement of XIAP level in pancreatic cancer tissue. The specimens were fixed in $10 \%$ formalin and embedded in paraffin. Five serial $4-\mu \mathrm{m}$ sections were cut from the tissue blocks. The deparaffinized sections were stained with the anti-XIAP antibody (BD Biosciences, Franklin Lakes, NJ, USA) at a dilution of $1: 100$ overnight at $4^{\circ} \mathrm{C}$ followed by incubation with a biotinylated secondary anti-mouse IgG antibody for $1 \mathrm{~h}$ at room temperature. The sections were subsequently incubated with horseradish peroxidase (HRP)-conjugated streptavidin and were developed using 3,3'-diaminobenzidine 
(DAB). XIAP staining was performed using the methods previously described. Grading was performed by two pathologists with no background on the clinical data of patients (9). XIAP staining was scored as: $0,0-25 \% ; 1, \geq 25-50 \% ; 2, \geq 50-75 \%$ and $3, \geq 75 \%$.

Statistical analysis. Statistical analysis was performed using the $\chi^{2}$ test to determine the XIAP expression frequency between normal and pancreatic cancer tissues. Spearman's correlation analysis was used to assess the correlation between XIAP expression and patient clinical data. Post-operative survival was estimated using the Kaplan-Meier method and differences between survival curves were compared using the log-rank test. Multivariate survival analysis was performed using the Cox proportional hazards model. Statistical analyses were performed using the SPSS 13.0 software (SPSS, Inc, Chicago, IL, USA). $\mathrm{P}<0.05$ was considered to indicate a statistically significant difference.

\section{Results}

XIAP staining assessment in normal and pancreatic cancer tissues. XIAP was mostly located in the cytoplasm, showing diffuse distribution. The cytoplasm of positive cells was stained yellow or brown (Fig. 1). Compared to the lower XIAP staining frequency in normal pancreatic tissue observed in 7 of 14 cases, XIAP was expressed in 48 of 54 pancreatic cancer tissues $(\mathrm{P}=0.001)$. These findings demonstrate that XIAP expression varied in normal and pancreatic cancer tissues.

Correlation between XIAP expression and post-operative survival. A total of 54 patients including 18 of stage I, 19 of stage II, 4 of stage III and 13 of stage IV were included in this study and a review was conducted every 3 months between 2003 and 2009. Two patients were lost during the follow-up period and the remaining 52 patients were included in the subsequent analysis. Forty-five patients had succumbed to the disease by the end of the follow-up period. The median follow-up time for the 52 patients was $14.39 \pm 13.37$ months. Adjuvant chemo- or radiation therapy was not administered following carcinoma resection. Data collected during the follow-up were analyzed for clinical characteristics as described in Materials and methods. No statistically significant difference was observed for gender or age, while borderline statistically significant difference was observed for tumor size and histological grade ( $\mathrm{P}=0.069$ and 0.079 , respectively). A significant difference was observed in the TNM classification and tumor invasion status, $\mathrm{P}<0.000$, respectively (Table I).

XIAP expression levels were graded with a score of 0-3. Patients were divided into two subgroups for survival analysis, with a scored of 0 and 1 for the low-expression group and a score of 2 and 3 for the high-expression group. The cumulative survival rate was plotted as a Kaplan-Meier curve (Fig. 2), and the log-rank test demonstrated a statistically significant difference $(\mathrm{P}=0.027)$ between the low- and high-expression groups, demonstrating the usefulness of XIAP levels in pancreatic cancer prognosis.

Effect of XIAP expression on the predictive power of traditional clinical characteristics. A multivariate analysis,
Table I. Univariate analysis of clinical characteristics associated with post-operative survival in pancreatic cancer patients.

\begin{tabular}{lccc}
\hline Characteristics & $\begin{array}{c}\text { No. of } \\
\text { cases }\end{array}$ & $\begin{array}{c}\text { Survival } \\
\text { rate (\%) }\end{array}$ & P-value \\
\hline Gender & & & 0.810 \\
$\quad$ Male & 34 & 20.6 & \\
$\quad$ Female & 20 & 5.0 & \\
Age (years) & & & 0.883 \\
$\quad<60$ & 21 & 14.3 & \\
$\geq 60$ & 33 & 15.2 & \\
Diameter of tumor (cm) & & & \\
$\quad<5$ & 26 & 19.2 & \\
$\geq 5$ & 28 & 10.7 & \\
TNM classification & & & \\
I-II & 37 & 18.9 & \\
III-IV & 17 & 5.9 & \\
Histological grade & & & \\
$\quad$ Well & 37 & 16.2 & \\
Moderately and poorly & 17 & 11.8 & \\
Invasion or metastasis & & & \\
Yes & 30 & 23.3 & \\
No & 24 & 4.2 & \\
\hline
\end{tabular}

TNM, tumor-node-metastasis; well, well-differentiated; moderately and poorly, moderately- and poorly-differentiated.

A

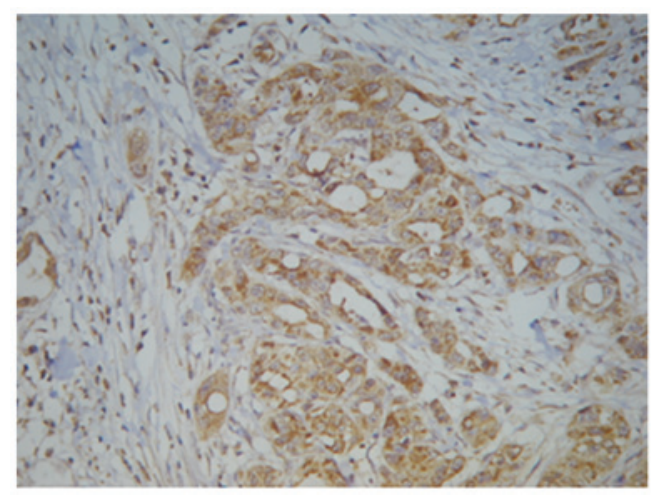

B

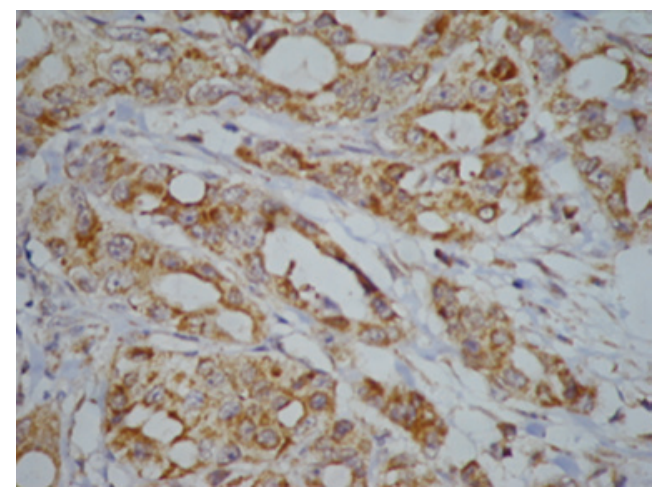

Figure 1. Immunohistochemical detection of XIAP expression in pancreatic carcinoma. Cytoplasmic expression of XIAP in $>75 \%$ cells. Magnification: (A) $\mathrm{x} 200$, (B) $\mathrm{x} 400$. 
Table II. Multiple analysis of prognostic factors associated with post-operative survival in pancreatic cancer patients using the COX proportional hazard model.

\begin{tabular}{lccc}
\hline Characteristics & Relative risk & 95\% CI & P-value \\
\hline Age & 1.004 & $0.978-1.030$ & 0.770 \\
Gender & 1.131 & $0.515-2.481$ & 0.759 \\
Diameter of tumors & 0.918 & $0.829-1.018$ & 0.105 \\
Histological grade & 0.675 & $0.353-1.290$ & 0.234 \\
TNM stage & 1.652 & $1.014-2.694$ & 0.044 \\
Invasion or metastasis & 2.229 & $0.805-6.167$ & 0.123 \\
XIAP expression & 1.771 & $1.099-2.852$ & 0.019 \\
\hline
\end{tabular}

CI, confidence interval; TNM, tumor-node-metastasis; XIAP, $\mathrm{X}$-linked inhibitor of apoptosis protein.

Table III. Comparison of XIAP expression with clinical characteristics in cancer tissue.

\begin{tabular}{|c|c|c|c|c|c|c|}
\hline \multirow[b]{2}{*}{ Characteristics } & \multirow[b]{2}{*}{ No. } & \multicolumn{4}{|c|}{ XIAP expression } & \multirow[b]{2}{*}{ P-value } \\
\hline & & 0 & 1 & 2 & 3 & \\
\hline \multicolumn{7}{|l|}{ Gender } \\
\hline Male & 34 & 4 & 3 & 15 & 12 & 0.406 \\
\hline Female & 20 & 2 & 4 & 9 & 5 & \\
\hline \multicolumn{7}{|l|}{ Age (years) } \\
\hline$<60$ & 21 & 3 & 5 & 8 & 5 & 0.116 \\
\hline$\geq 60$ & 33 & 3 & 2 & 16 & 12 & \\
\hline \multicolumn{7}{|c|}{ Tumor diameter $(\mathrm{cm})$} \\
\hline$<5$ & 26 & 5 & 4 & 9 & 8 & 0.268 \\
\hline$\geq 5$ & 28 & 1 & 3 & 15 & 9 & \\
\hline \multicolumn{7}{|c|}{ TNM classification } \\
\hline $\mathrm{I}+\mathrm{II}$ & 37 & 5 & 6 & 16 & 10 & 0.152 \\
\hline III+IV & 17 & 1 & 1 & 8 & 7 & \\
\hline \multicolumn{7}{|c|}{ Invasion or metastasis } \\
\hline No & 30 & 6 & 4 & 13 & 7 & 0.040 \\
\hline Yes & 24 & 0 & 3 & 11 & 10 & \\
\hline \multicolumn{7}{|c|}{ Histological grade } \\
\hline Well & 12 & 2 & 2 & 6 & 2 & 0.014 \\
\hline Moderately & 25 & 3 & 5 & 11 & 6 & \\
\hline Poorly & 17 & 1 & 0 & 7 & 9 & \\
\hline
\end{tabular}

XIAP, X-linked inhibitor of apoptosis protein; TNM, tumornode-metastasis; well, well-differentiated; moderately, moderatelydifferentiated; poorly, poorly-differentiated.

including the predictors mentioned above, using the Cox proportional hazard method was used to evaluate the effect of XIAP expression on the predictive power of traditional clinical parameters. The analysis demonstrated that the XIAP level and TNM classification were independent risk factors for the post-operative survival rate of pancreatic cancer patients

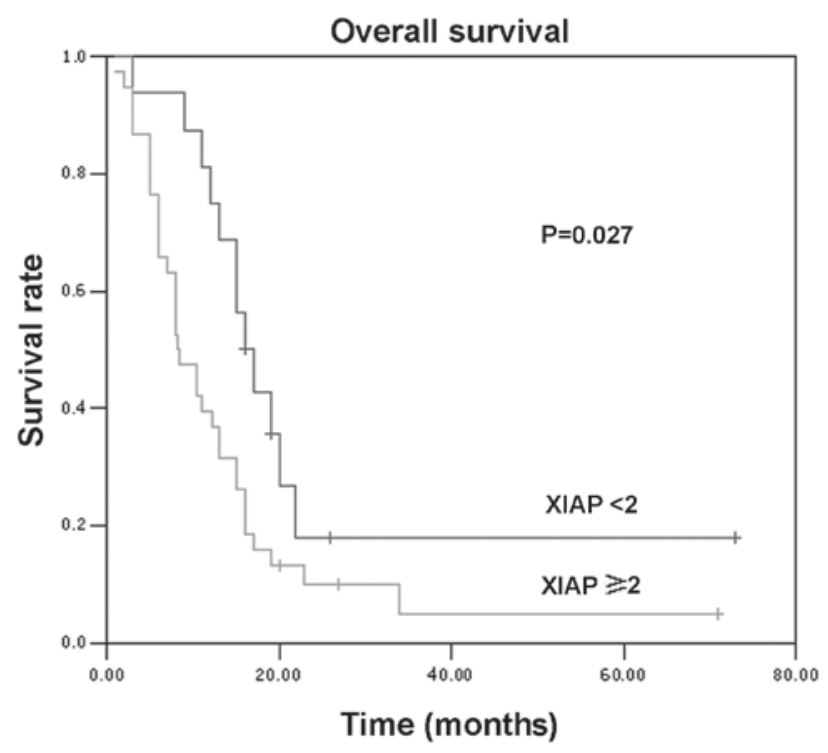

Figure 2. Survival curves for OS of various subgroups based on different levels of XIAP expression. A significant correlation pattern was detected in patients when categorized into subgroups of high $(\geq 2)$ and low $(<2)$ XIAP expression $(\mathrm{P}=0.027)$.

(Table II). Of the predictors, XIAP expression status was strongly associated with the cancer survival rate, at a hazard ratio of 1.771 (95\% CI, 1.099-2.852). Results of the univariate and multivariate analyses suggested that the XIAP level in pancreatic cancer tissues constitutes one of the most significant predictors of the post-operative survival rate of pancreatic cancer patients.

Correlation of clinical characteristics and XIAP levels. The correlation of clinical characteristics and XIAP levels was investigated in pancreatic cancer patients using the Spearman's correlation analysis (Table III). Gender, age, tumor size and TNM classification were not correlated with XIAP levels, while tumor invasion status and histological grade were significantly correlated with XIAP expression. The data suggest that XIAP is able to modify tumor development in combination with other predictors.

\section{Discussion}

In the present study, we used immunohistochemical methods with a monoclonal antibody against XIAP with paraffinembedded pancreatic tissue sections for the assessment of this apoptosis inhibitor in pancreatic cancer patients. Consistent with the findings of previous studies, different expression frequencies were found in normal and pancreatic cancer tissues (7). XIAP expression was also found to be associated with the outcome of pancreatic cancer patients. Similar results have also been demonstrated in other types of cancer where XIAP overexpression was correlated with worse prognosis (10-12). XIAP levels constituted a risk factor for pancreatic carcinogenesis and a good marker for predicting the outcome of post-operative pancreatic cancer patients.

IAP is a family of endogenous caspase inhibitors that share a common baculoviral IAP repeat (BIR) domain. 
XIAP carrying three functional domains including BIR, linker and Ring-finger domains, potentially constitutes the best-characterized IAP proteins with respect to its structure and biochemical mechanisms. XIAP exhibits its effect on resistance to apoptosis by inhibiting caspases-3, -7 and -9 , but not caspases-1, $-6,-8$ and $-10(7,13,14)$. XIAP is a potential target for pancreatic carcinoma treatment due to the fact that XIAP blocks apoptosis at the core of the apoptotic machinery. XIAP inhibitors increase apoptosis sensitivity in pancreatic carcinoma cells (7), while XIAP knockdown inhibits pancreatic cancer cell proliferation in vitro and in vivo (15). XIAP overexpression shortens the survival time of pancreatic cancer patients probably by modifying the resistance to apoptosis and the proliferation capacity of pancreatic carcinoma cells.

The clinical significance of XIAP expression was investigated in this study. The expression of XIAP in pancreatic cancer was not significantly correlated with gender, age, tumor size or TNM classification. However, increased XIAP expression was detected in less differentiated pancreatic carcinoma tissues, confirming the data provided by Jian et al (16). Furthermore, the prevalence of XIAP expression in cases with a tumor invasion status suggested a correlation of XIAP expression with a more aggressive phenotype of pancreatic cancer.

The balance of anti- to pro-apoptotic regulators has been shown to control the relative sensitivity or resistance of various cells to apoptotic stimuli. Smac/DIABLO inhibits XIAP and enhances apoptosis sensitivity by binding with the BIR structure of human IAP family genes. An inverse correlation between XIAP expression and Smac/DIABLO mitochondrial release has been observed following apoptosis induction in colon cancer cells, lymphoma cells and keratinocytes (17-19). The delicate balance between XIAP and Smac/DIABLO expression was gradually disturbed in certain types of cancer, resulting in a relative increase of the anti-apoptotic XIAP levels exceeding the pro-apoptotic Smac/DIABLO levels, a fact which may be responsible for the carcinogenesis and marked resistance to apoptosis of these types of cancer $(10,20)$. In this study, XIAP expression was also found to have a significant inverse correlation with Smac expression in pancreatic cancer tissue $(\mathrm{P}=0.002)$, however, Smac expression was not associated with pancreatic carcinoma outcome (data not shown).

In conclusion, to the best of our knowledge, the correlation of XIAP expression with prognosis was analyzed for the first time in patients with pancreatic cancer. Data in this study obtained by univariate and multivariate analysis showed that patients in the high XIAP expression subgroups exhibited a significantly shorter overall survival. Additional studies are required to determine the key factors involved in the process of XIAP-related resistance to apoptosis.

\section{Acknowledgements}

This study was supported by the Natural Science Foundation of the Hebei Province (no. C 2006000856).

\section{References}

1. Li D, Xie K, Wolff R and Abbruzzese JL: Pancreatic cancer. Lancet 363: 1049-1057, 2004.

2. Hidalgo M: Pancreatic cancer. N Engl J Med 362: 1605-1617, 2010.
3. Schneider G, Siveke JT, Eckel F and Schmid RM: Pancreatic cancer: basic and clinical aspects. Gastroenterology 128: 1606-1625, 2005.

4. Gukovskaya AS and Pandol SJ: Cell death pathways in pancreatitis and pancreatic cancer. Pancreatology 4: 567-586, 2004.

5. Fulda S and Debatin KM: Extrinsic versus intrinsic apoptosis pathways in anticancer chemotheraphy. Oncogene 25: 4798-4811, 2006.

6. Neesse A, Gress TM and Michl P: Therapeutic targeting of apoptotic pathways: novel aspects in pancreatic cancer. Curr Pharm Biotechnol: May 2, 2011 (Epub ahead of print).

7. Vogler M, Walczak H, Stadel D, Haas TL, Genze F, Jovanovic M, Bhanot U, Hasel C, Möller P, Gschwend JE, Simmet T, Debatin KM and Fulda S: Small molecule XIAP inhibitors enhance TRAIL-induced apoptosis and antitumor activity in preclinical models of pancreatic carcinoma. Cancer Res 69: 2425-2434, 2009.

8. Sobin LH and Wittekind $\mathrm{CH}$ (eds): International Union Against Cancer (UICC), TNM Classification of Malignant Tumors. 6th edition. John Wiley \& Sons, New York, 2002.

9. Wang J, Liu Y, Ji R, Gu Q, Zhao X, Liu Y and Sun B: Prognostic value of the $X$-linked inhibitor of apoptosis protein for invasive ductal breast cancer with triple-negative phenotype. Hum Pathol 41: 1186-1195, 2010.

10. Yan Y, Mahotka C, Heikaus S, Shibata T, Wethkamp N, Liebmann J, Suschek CV, Guo Y, Gabbert HE, Gerharz CD and Ramp U: Disturbed balance of expression between XIAP and Smac/DIABLO during tumour progression in renal cell carcinomas. Br J Cancer 91: 1349-1357, 2004.

11. Augello C, Caruso L, Maggioni M, Donadon M, Montorsi M, Santambrogio R, Torzilli G, Vaira V, Pellegrini C, Roncalli M, Coggi G and Bosari S: Inhibitors of apoptosis proteins (IAPs) expression and their prognostic significance in hepatocellular carcinoma. BMC Cancer 9: 125, 2009.

12. Nakagawa Y, Abe S, Kurata M, Hasegawa M, Yamamoto K, Inoue M, Takemura T, Suzuki K and Kitagawa M: IAP family protein expression correlates with poor outcome of multiple myeloma patients in association with chemotherapy-induced overexpression of multidrug resistance genes. Am J Hematol 81: 824-831, 2006.

13. Deveraux QL, Takahashi R, Salvesen GS and Reed JC: X-linked IAP is a direct inhibitor of cell death proteases. Nature 388: 300-304, 1997.

14. Riedl SJ, Renatus M, Schwarzenbacher R, Zhou Q, Sun C, Fesik SW, Liddington RC and Salvesen GS: Structural basis for the inhibition of caspase-3 by XIAP. Cell 104: 791-800, 2001

15. Jiang C, Tan T, Yi XP, Shen H and Li YX: Lentivirus-mediated shRNA targeting XIAP and survivin inhibit SW1990 pancreatic cancer cell proliferation in vitro and in vivo. Mol Med Rep 4: 667-674, 2011.

16. Jian ZY, Li YX, Li XG and Chen JY: Expression and significance of XIAP in pancreatic carcinoma tissues. Chin J Gen Surg 14: 385-387, 2005 (In Chinese).

17. Tillman DM, Izeradjene K, Szucs KS, Douglas L and Houghton JA: Rottlerin sensitizes colon carcinoma cells to tumor necrosis factor-related apoptosis-inducing ligand-induced apoptosis via uncoupling of the mitochondria independent of protein kinase C. Cancer Res 63: 5118-5125, 2003.

18. Chow KU, Nowak D, Boehrer S, Ruthardt M, Knau A, Hoelzer D, Mitrou PS and Weidmann E: Synergistic effects of chemotherapeutic drugs in lymphoma cells are associated with down-regulation of inhibitor of apoptosis proteins (IAPs), prostate-apoptosis-response-gene 4 (Par-4), death-associated protein (Daxx) and with enforced caspase activation. Biochem Pharmacol 66: 711-724, 2003.

19. Takasawa R and Tanuma S: Sustained release of Smac/DIABLO from mitochondria commits to undergo UVB-induced apoptosis. Apoptosis 8: 291-299, 2003.

20. Zhang Y, Zhu J, Tang Y, Li F, Zhou H, Peng B, Zhou C and Fu R: X-linked inhibitor of apoptosis positive nuclear labeling: a new independent prognostic biomarker of breast invasive ductal carcinoma. Diagn Pathol 6: 49, 2011. 